\title{
A descentralização do sistema de saúde no Brasil ${ }^{1}$
}

Nilson do Rosário Costa

Pedro Luís Barros Silva

José Mendes Ribeiro

\section{Introdução}

Os efeitos imprevistos do processo de descentralização da provisão de atenção à saúde, ocorrido na década de 90, têm gerado um grande número de estudos de avaliação de política pública e análises indutivas de políticas setoriais das organizações internacionais. Esses trabalhos apresentam como traço comum o uso, excessivamente abrangente, do conceito de descentralização, que tem sido utilizado para nomear qualquer política de flexibilização da gestão pública promovida pelos governos centrais. Os diferentes modos de flexibilização da gestão central aparecem nas políticas de desconcentração, devolução e delegação de provisão de serviços sociais para níveis subnacionais e além do processo de privatização.

As iniciativas de delegação, devolução ou desconcentração podem, em certas circunstâncias, ser incluídas no processo de descentralização. O mesmo não ocorre com relação à política de privatização, tanto pela sua singularidade macroeconômica como pelo seu impacto sobre a reforma do Estado, merecendo assim um tratamento analítico específico (Via i Redons \& McDonagh, 1999).

Considerando-se essas questões, utiliza-se nesse texto o conceito de descentralização como processo de transferência de autoridade ou poder decisório no financiamento e gestão do nível nacional para níveis subnacionais. Esse processo chega a definir a responsabilidade pela tomada de decisão ao menor nível organizacional possível. Nesse sentido, pode-se afirmar, para a experiência brasileira, que os processos de flexibilização administrativa e terceirização também fazem parte do processo de descentralização. Esses processos oferecem um amplo campo de análise

\footnotetext{
Nilson do

Rosário Costa, sociólogo, doutor em planejamento urbano e pesquisador titular da ENSP/ FIOCRUZ/RJ.

Pedro Luís Barros Silva, administrador público, doutor em ciência política, professor do Instituto de Economia e coordenador do NEPP/ UNICAMP/SP. José Mendes Ribeiro, médico, doutor em ciências e pesquisador adjunto da ENSP/ FIOCRUZ/RJ
} 
da mudança das funções públicas que não serão abordados neste texto,

que está focado no aspecto da descentralização diretamente associada às relações intragovernamentais.

A rápida difusão da agenda da descentralização da atenção à saúde no Brasil tem uma relação direta com a percepção generalizada sobre o déficit de efetividade e de responsabilização da política social, agravado pela baixa definição dos papéis para os níveis de governo que atuam no setor (União, estados e municípios).

A descentralização tem como meta a definição precisa desses papéis, pressupondo, ademais, que a provisão direta e exclusiva, ao nível da gestão municipal, permite maior controle de qualidade pelo cidadão/cliente dos serviços prestados e/ou contratados pelo setor público.

Embora os indicadores sociais tenham apresentado importantes progressos no Brasil ao longo das décadas de 80 e 90, em razão da queda da mortalidade infantil, da expansão do consumo de água potável e da ampliação do acesso na atenção à saúde (PNUD, 1996), permanece em debate o problema da baixa efetividade das políticas sociais.

A situação social brasileira é bastante crítica se comparada a países com renda per capita semelhante (World Bank, 1988). Essa percepção tem exigido, além da sustentabilidade dos gastos públicos e melhor padrão de distribuição da renda interna, a melhoria específica de desempenho do sistema de proteção social.

O processo de descentralização da provisão dos serviços de saúde, motivado por essa percepção do déficit de efetividade, tem gerado mudanças importantes na configuração do subsistema público universalista, denominado pela Constituição de 1988 como Sistema Único de Saúde (SUS), redefinindo os papéis da União (Governo Federal), estados e municípios na oferta de atenção à saúde.

A rapidez da descentralização da política de saúde tem sido um resultado bem sucedido das demandas dos gestores locais e regionais por autonomia decisória e responsabilidade pela provisão direta de serviços sociais, originadas no processo de redemocratização das décadas de 70 e 80. Entre os pontos críticos do desenho institucional da provisão de proteção social foram assinalados:

- a centralização decisória em nível federal, mediante a desapropriação dos instrumentos de ação social de estados e municípios;

- bloqueio à participação social e política no processo decisório, com a supressão das formas de controle social;

- a fragmentação institucional que impossibilitava a constituição de uma política social integrada;

- fechamento dos canais de acesso da população aos bens e serviços sociais. 
A agenda da redemocratização tem buscado, na ampliação das responsabilidades da gestão local, uma alternativa para o desenvolvimento de mecanismos de controle sobre o gasto público e de ampliação do acesso às políticas sociais.

A Constituição de 88 fortaleceu as demandas localistas, promovendo uma repartição do bolo fiscal, que favoreceu os interesses locais e regionais em detrimento do governo central (União). Como mostra a Tabela 1, de 1980 a 1993, os municípios e os estados passaram de $31 \%$ para $42 \%$ de participação nas receitas disponíveis pela federação. No mesmo período, os municípios registraram um crescimento específico de participação de $9 \%$ para $16 \%$ das receitas públicas.

Tabela 1: Evolução da receita disponível por esfera de governo (em porcentagem). Brasil, 1980/88/93

\begin{tabular}{c|c|c|c}
\hline Ano & União (\%) & Estados (\%) & Municípios (\%) \\
\hline 1980 & 69 & 22 & 9 \\
\hline 1988 & 62 & 27 & 11 \\
\hline 1993 & 58 & 26 & 16 \\
\hline
\end{tabular}

Fonte: Afonso \& Silva (1995).

Essa repartição das receitas nacionais sustentou o crescimento relativo da gestão municipal, tanto na administração pública nacional como em relação à execução da maioria dos programas sociais. $\mathrm{O}$ aumento de responsabilidade municipal motivou também o crescimento da arrecadação tributária própria dos municípios, apresentando um incremento médio anual duas vezes maior que os tributos estaduais e cerca de três vezes o dos federais após a Constituição de 88, como mostra a tabela abaixo.

Tabela 2: Desempenho da arrecadação tributária por nível de governo (em porcentagem). Brasil, 1988 a 1997

\begin{tabular}{c|c|c|c}
\hline Ano & União $(\boldsymbol{\%})$ & Estados $(\boldsymbol{\%})$ & Municípios (\%) \\
\hline 1988 & 100 & 100 & 100 \\
\hline 1989 & 106 & 119 & 102 \\
\hline 1990 & 121 & 130 & 130 \\
\hline 1991 & 101 & 128 & 189 \\
\hline 1992 & 101 & 117 & 162 \\
\hline 1993 & 110 & 108 & 144 \\
\hline 1994 & 122 & 133 & 155 \\
\hline 1995 & 129 & 148 & 215 \\
\hline 1996 & 137 & 163 & 284 \\
\hline 1997 & 144 & 165 & 290 \\
\hline
\end{tabular}

Fonte: Afonso, J.R.R. et al. "Municípios, Arrecadação e Administração Tributária: Quebrando Tabus", Rio de Janeiro, BNDES, 1999, p.6. No período 1988/1997 não foram consideradas as transferências federais. 


\section{A descentralização da saúde na Constituição de 1988 e a responsabilidade municipal na provisão dos serviços}

A Constituição Federal de 1988 foi um momento inaugural da orientação descentralizadora para o setor saúde como uma alternativa aceitável para a reversão da baixa qualidade da gestão pública na área social, para a redefinição das prioridades das ações estatais destinadas ao atendimento das necessidades da população e para a ampliação da autonomia de gestão das autoridades públicas locais.

O capítulo referente à saúde consolidou as tendências na gestão pública setorial de:

- reconhecimento da saúde como direito social, assegurado pelo acesso universal e igualitário às ações e serviços de promoção, proteção e prevenção da saúde (Art. 196);

- estabelecimento de um novo paradigma para a ação estatal na área, ao determinar o atendimento integral, a descentralização políticoadministrativa e a participação comunitária (Art.198, I, III);

- comprometimento financeiro pela criação de um orçamento público da seguridade social que sustenta um conjunto integrado de ações capaz de "assegurar os direitos relativos à saúde, à previdência e à assistência social" (Art.194);

- unificação da ação pública pela definição do SUS como instância única da gestão pública responsável pela provisão universal, gratuita e integral de ações e serviços de saúde para a promoção, proteção e recuperação da saúde (Art. 196 e 198).

A tendência à descentralização setorial, mediante o processo de redistribuição de capacidade decisória e de recursos entre esferas de governo, é traduzida pela definição do município como o único ente federativo ao qual é atribuído a missão constitucional de prestar serviço de atendimento à saúde da população. À União e aos estados cabem prover a cooperação técnica e financeira necessária ao exercício desse encargo (Art. 30, VIII).

A lei $\mathrm{n}^{\circ} 8.080$ de setembro de 1990 ratifica as diretrizes constitucionais ao atribuir à direção municipal do SUS a competência de "planejar, organizar, controlar e avaliar as ações de saúde, e gerir e executar os serviços de saúde", bem como participar do "planejamento, programação e organização da rede regionalizada e hierarquizada do SUS, em articulação com sua direção estadual" (Art.18, I e II).

Essas mudanças de titularidade da gestão municipal induziram ao crescimento do papel dos governos locais na provisão da atenção, reduzindo ao longo da década de 80 as funções da União na oferta direta de serviços de atenção à saúde, e ampliando as suas atividades de negociação na 
alocação de recursos e regulação de desempenho. Essa redefinição de

funções indica que o SUS tem implementado, de modo acelerado, a separação entre financiamento e provisão, pelo menos nas relações entre os níveis de governo (World Bank, 1998).

Os dados da Tabela 3 sugerem que o incentivo à maior autonomia na gestão administrativa e financeira local resultou em expressivo crescimento de estabelecimentos sob gestão municipal: essa evoluiu, entre os anos 81 a 92 , de $22 \%$ para $69 \%$, enquanto os estabelecimentos sob gestão federal regrediram de $28 \%$ para apenas $5 \%$, e os sob gestão estadual de $50 \%$ para $26 \%$.

Tabela 3: Evolução do número de estabelecimentos do setor público de saúde por esfera administrativa. Brasil, 1981/88/92

\begin{tabular}{c|l|c|c|c}
\hline Ano & Federal & \multicolumn{1}{c|}{ Estadual } & \multicolumn{1}{c}{ Municipal } & \multicolumn{1}{c}{ Total } \\
\hline 1981 & $3.764(28 \%)$ & $6.890(50 \%)$ & $2.961(22 \%)$ & $13.615(100 \%)$ \\
\hline 1988 & $1.978(9 \%)$ & $10.643(50 \%)$ & $8.851(41 \%)$ & $21.472(100 \%)$ \\
\hline 1992 & $1.387(5 \%)$ & $7.043(26 \%)$ & $18.662(69 \%)$ & $27.092(100 \%)$ \\
\hline
\end{tabular}

Fonte: IBGE/AMS (1981/88/92).

O aumento da responsabilidade municipal na provisão de serviços de saúde é também comprovado segundo os critérios regionais, como indica a Tabela 4. A evolução da responsabilidade municipal é especialmente evidente nas regiões Sul e Sudeste, comprovada pela proporção de 82,7\% e $84,9 \%$ do total de estabelecimentos públicos, enquanto a gestão federal $(2,2 \%$ e $2,5 \%)$ e a gestão estadual $(15,7 \%$ e $12,7 \%)$, respectivamente, tornaram-se quase residuais.

Tabela 4: Distribuição percentual dos estabelecimentos do setor público de saúde por grandes regiões (em porcentagem). Brasil, 1992

\begin{tabular}{l|c|c|c|c}
\hline Brasil e grandes regióes & Federal (\%) & Estadual (\%) & Municipal (\%) & Total (\%) \\
\hline Norte & 11,5 & 35,5 & 53,0 & 100 \\
\hline Nordeste & 7,4 & 41,5 & 51,1 & 100 \\
\hline Sudeste & 2,5 & 12,7 & 84,8 & 100 \\
\hline Sul & 2,2 & 15,1 & 82,7 & 100 \\
\hline Centro-Oeste & 4,8 & 32,2 & 63,0 & 100 \\
\hline Brasil & 5,1 & 26,0 & 68,9 & 100 \\
\hline
\end{tabular}

Fonte: IBGE-AMS (1992). 
A mesma tendência ao fortalecimento municipal é ratificada pela evolução do emprego no setor público da saúde no período 1980-92, que registrou a inversão de papéis entre os níveis federal e municipais. O município oferece, em 1992, 43,7\% dos empregos na saúde, apesar de possuir apenas $14,9 \%$ em 1988. O nível federal decresceu de 42,6\%, em 1988, para 14, 9\%, em 1992, conforme tabela abaixo.

Tabela 5: Evolução e participação relativa do emprego no setor público de saúde, por esfera administrativa. Brasil, 1981/92

\begin{tabular}{c|c|c|c|c}
\hline Ano & Federal & Estadual & Municipal & Total \\
\hline 1981 & $113.297(43 \%)$ & $109.373(41 \%)$ & $43.086(16 \%)$ & $265.956(100 \%)$ \\
\hline 1992 & $80.298(15 \%)$ & $222.139(41 \%)$ & $235.321(44 \%)$ & $537.688(100 \%)$ \\
\hline P $\%$ & $-2,6 \%$ & $6 \%$ & $13,9 \%$ & $5,6 \%$ \\
\hline
\end{tabular}

Sendo $\mathrm{P} \%$ = taxa média de crescimento geométrico anual.

Fonte: IBGE-AMS (1981/92).

Os dados comparativos da distribuição de estabelecimentos de saúde entre prestadores — federal, estadual, privado e municipal — indicam que o crescimento do setor público (SUS), vis-à-vis o dinamismo do setor privado de seguro-saúde e contratado pelo setor público na década de 80 , deveu-se ao crescimento do papel do município no contexto nacional e do nível estadual no Norte, Nordeste e Centro-Oeste (IBGE, 1993).

Como mostra a Tabela 6, enquanto a participação de estados e da União diminuiu, os municípios fortaleceram a participação na gestão direta de estabelecimentos de saúde de 32\%, em 88, para 38\%, em 92, acompanhando, em parte, a impressionante evolução do setor privado, que aumentou a participação de $22 \%$ para $45 \%$, entre esses anos.

Tabela 6: Evolução dos estabelecimentos de saúde por natureza jurídica (em porcentagem). Brasil, 1988/92

\begin{tabular}{l|c|c}
\hline & $\mathbf{1 9 8 8}(\boldsymbol{\%})$ & $\mathbf{1 9 9 2}(\boldsymbol{\%})$ \\
\hline Públicos & 77,7 & 44,5 \\
\hline Federal & 7,2 & 2,8 \\
\hline Estadual & 38,5 & 14,2 \\
\hline Municipal & 32,0 & 37,6 \\
\hline Privados & 22,3 & 45,5 \\
\hline Totais & 100 & 100 \\
\hline
\end{tabular}

Fonte: IBGE-AMS (1988/92). 


\section{As Normas Operacionais \\ Básicas e a nova estrutura da gestão pública na saúde}

A descentralização na década de 90 foi impulsionada e regulamentada através das Normas Operacionais Básicas (NOB), que são orientações específicas e pactuadas, emanadas pelo Ministério da Saúde (MS) e aceitas pelas representações nacionais dos secretários municipais e estaduais de saúde, visando a repartição orçamentária entre níveis de governo, a atribuição de titularidade para a gestão e a organização do modelo assistencial. A primeira norma vigorou em 1991, estabelecendo regras de transferências de recursos entre a União, a habilitação para a provisão direta dos serviços de saúde e as competências entre os três níveis de governo da federação brasileira (União, Estados e Distrito Federal, e Municípios).

A NOB 1/91 introduziu o conceito de municípios e estados habilitados ao SUS, mediante comprometimento com a gestão direta de serviços. Nesse sentido, previa a formação de um fundo setorial e o funcionamento de conselhos comunitários.

A NOB 1/93 representou um instrumento mais efetivo de indução do processo de descentralização política ao criar as Comissões Intergestoras Bipartites (CIB) nos estados (por representação paritária entre estados e municípios) e as Comissões Intergestoras Tripartites (CIT), compostas por representantes dos governos federal, estaduais e municipais.

Além disso, essa norma introduziu uma escala aplicável a estados e municípios habilitados — municípios com condição de gestão incipiente, parcial ou semiplena - segundo níveis de comprometimento e de estrutura administrativa , que definiu o modo como as transferências dos recursos da União ocorreram no biênio 93/94.

A NOB 1/93 produziu uma modulagem institucional do processo de descentralização e de responsabilização das instâncias de governo em relação à saúde. Representou, assim, um divisor de águas na política social brasileira dos anos 90 ao formatar um processo de normalização das relações intragovernamentais, criando regras de transferências de recursos, mecanismos de controle e avaliação, e definição de etapas não-seqüenciais de transferência para a gestão local de serviços.

A NOB 1/93 desenhou três situações transacionais para a gestão do sistema de saúde nas esferas sub-nacionais já mencionadas: situação transacional incipiente, transacional parcial e transacional semiplena, representando etapas progressivas de titularidade governativa.

Para ter condições de assumir um desses status na gestão setorial, os governos locais deveriam atingir uma capacidade estatal associada a variáveis de responsabilização e controle externo das suas decisões. Por exemplo, na situação transacional de gestão semiplena, foram demandados 
compromissos inusitados nas relações intragovernamentais, historicamente orientadas por relações de baixa responsabilização e déficit de auditagem. Para obter a situação de gestão semiplena na saúde, os municípios assumiram os compromissos de:

- ampliar a capacidade gerencial de planejamento, avaliação e controle dos serviços de saúde;

- instituir conselho de saúde;

- criar um fundo de saúde;

- desenvolver um plano de saúde;

- elaborar relatório de gestão para efeito de auditagem contendo os balancetes do Fundo de Saúde, as atas das reuniões do Conselho Municipal de Saúde, os dados sobre os gastos fiscais próprios destinados à saúde;

- informar sobre os recursos organizacionais locais para auditar os gastos com serviços ambulatoriais e hospitalares contratados.

$\mathrm{O}$ prêmio para a capacidade de responder a esses requisitos foi a obtenção de autonomia governativa municipal em relação a ações e serviços de saúde. As secretarias municipais de saúde ganharam:

- a titularidade sobre autorização de credenciamento, descrendeciamento, controle e avaliação dos serviços ambulatoriais e hospitalares privados e filantrópicos;

- a gestão da totalidade das cotas de Autorização de Internação Hospitalar (AIH);

- gerenciamento da rede ambulatorial;

- a incorporação das ações de vigilância epidemiológica e sanitária à rede de serviços.

Esse aumento de responsabilidade incentivado pela NOB 1/93 serviu como mecanismo de aceleração da adesão municipal às novas funções propostas do SUS, gerando o seguinte quadro:

- de um total de 4.973 municípios existentes em 1996 no País, $65,05 \%$ enquadravam-se em uma das modalidades de gestão proposta pelo SUS: 49,38\% estavam habilitados em gestão incipiente; na modalidade de gestão parcial enquadravam-se $12,85 \%$ dos municípios; e na habilitação semiplena, 2,86\%;

- para os municípios que assumiram a condição de gestão semiplena transferiram-se, direta e automaticamente, aproximadamente $25 \%$ dos recursos financeiros do Fundo Nacional de Saúde para a assistência ambulatorial e hospitalar;

- 11 capitais exercem a gestão semiplena da assistência à saúde: Região Norte: Belém; Região Nordeste: Natal, São Luís, João Pessoa, Recife, Teresina; Região Centro-Oeste: Campo Grande, Cuiabá; Região Sudeste: Belo Horizonte; Região Sul: Curitiba e Porto Alegre;

- nos estados onde os municípios-capitais estão na condição de gestão semiplena ocorreu um substancial deslocamento no controle dos 
recursos da saúde transferidos pela União à esfera municipal. Nos estados com capitais habilitadas, os gestores municipais em semiplena executaram em torno de $40 \%$ das transferências globais para os estados, excluindo-se $25 \%$, fator de reajuste repassado sobre os tetos globais (MS, 1997c).

O processo de descentralização através da NOB 1/93 foi descontinuado pela proposta da NOB 1/96, que tem como horizonte estratégico ampliar a responsabilidade do gestor municipal e estadual e induzir a um conjunto de mudanças no modelo assistencial, pelo fortalecimento da atenção básica e primária e das ações de saúde coletiva.

A NOB 1/96 simplificou o processo de responsabilização municipal proposto pela NOB 1/93, reduzindo as condições de habilitação a duas modalidades — Gestão Plena da Atenção Básica (GPAB) e Gestão Plena do Sistema Municipal (GPSM). Os principais objetivos da NOB 1/96 são:

- promover e consolidar a função municipal na gestão do poder público na atenção à saúde e redefinir as responsabilidades de estados, Distrito Federal e União;

- estabelecer um novo pacto federativo em relação à responsabilidade sanitária;

- reorganizar o modelo assistencial, dando ao município a responsabilidade pela gestão e execução direta da atenção básica à saúde;

- assegurar que as transferências intragovernamentais sejam fundo a fundo, reduzindo as transferências para remuneração de serviços produzidos;

- fortalecer a gestão compartilhada entre os três níveis de governo através das CIT e CIB;

- estabelecer a direção única em cada nível de governo;

- fortalecer a função gestora das secretarias estaduais, com redefinição de responsabilidades para a coordenação do SUS em âmbito estadual;

- regulamentar as relações entre os sistemas municipais e desses com as instâncias estaduais e federais, destacando-se, como principal instrumento das relações entre governos, a Programação Pactuada e Integrada (PPI).

Em relação às transferências intragovernamentais, a NOB 1/96 cria um conjunto de instrumentos indutores de modelo assistencial ${ }^{2}$, onde se destacam:

- Piso Ambulatorial Básico (PAB), que é o montante de recursos financeiros destinados ao custeio dos procedimentos da atenção básica de responsabilidade tipicamente municipal: vacinação, consultas médicas, prénatal e atendimento ambulatorial. $\mathrm{O}$ valor das transferências é calculado pela multiplicação da população do município por um valor per capita nacional. Em março de 1999, o PAB garantia um mínimo de $\mathrm{R} \$ 10,00$ e um máximo de $\mathrm{R}$ \$ 18,00 per capita/ano para os municípios que investiram em ações de 
atendimento básico em saúde. O PAB é transferido diretamente, de forma regular e automática, ao município habilitado. Quando o município não é habilitado, o PAB é transferido diretamente ao respectivo estado. Para aqueles municípios que já apresentam gastos per capita superiores ao $\mathrm{PAB} /$ per capita nacional, a NOB/96 prevê um fator diferenciado de ajuste à sua implantação;

- o incentivo à adoção do Programa de Saúde da Família (PSF) e Programa de Agentes Comunitários (PACS) proporciona um adicional de transferência intragovernamental por grupo de população. O PSF assegura um recurso extra por equipe de profissionais da área médica implantada pelo município. As equipes são responsáveis pelo atendimento permanente de 3,5 mil pessoas;

- Fração Assistencial Especializada (FAE) é um montante de recursos definidos pela PPI, aprovada na CIB, referente a procedimentos ambulatoriais de média complexidade, medicamentos excepcionais, órteses e próteses ambulatoriais e tratamento fora do domicílio. A FAE é transferida, diretamente, aos municípios habilitados e fundo a fundo aos estados habilitados na Gestão Plena do Sistema Estadual (GPSE);

- Índice de Valorização de Resultados sobre as condições de saúde da população, transferidos para os estados;

- Piso Básico de Vigilância Sanitária e Índice de Valorização do Impacto em Vigilância para remunerar serviços produzidos nas ações de média e alta complexidade em vigilância sanitária e em programas desconcentrados.

Quando enquadrado na GPAB, o município passa a ser responsável pela atenção primária, ou seja, pela provisão, por intermédio de suas próprias unidades e/ou contratando serviços com o setor privado, de uma lista de atividades que incluem ações de alcance coletivo (como execução das ações básicas de vigilância sanitária) e procedimentos individuais de caráter ambulatorial, nas especialidades já referidas: clínica médica, ginecologia, obstetrícia, pediatria e pequenas cirurgias ambulatoriais.

Como exigência para atingir essa condição de gestão, o município deve comprovar, entre outras exigências formais:

- funcionamento do Conselho Municipal de Saúde;

- operação do Fundo Municipal de Saúde;

- existência do Plano Municipal de Saúde;

- capacidade administrativa para contratação, pagamento, controle e auditoria dos serviços sob sua gestão;

- comprometimento orçamentário com as contrapartidas previstas em lei;

- capacidade de desenvolvimento de ações de vigilância sanitária e epidemiológica. 
Para o município demandar a condição de GPSM, além dos requisitos anteriores para a habilitação na GPAB, deve apresentar: Plano Municipal de Saúde e relatório de gestão do ano anterior, aprovados pelo Conselho Municipal de Saúde.

Quando enquadrado na condição de GPSM de saúde, o gestor municipal tem autonomia para editar normas sobre o pagamento de serviços em seu território, inclusive alterando os valores de procedimentos médicos, tendo, contudo, a tabela nacional como referência mínima. A Tabela 7, a seguir, representa o balanço da descentralização até fins do ano de 1988. O município torna-se responsável pela programação, controle e remuneração dos serviços produzidos localmente, seja qual for o provedor.

\section{Tabela 7: Modalidades de incentivo do \\ Fundo Nacional de Saúde aos fundos estaduais e municipais (inclusões na NOB 1/96)}

\begin{tabular}{|c|c|c|}
\hline $\begin{array}{l}\text { Modalidades de incentivo } \\
\text { fundo a fundo da NOB } 1 / 96\end{array}$ & Implementado & $\begin{array}{l}\text { Não- } \\
\text { implementado }\end{array}$ \\
\hline PAB per capita (GPSM) & $\mathrm{X}$ & \\
\hline Teto financeiro global de município (GPSM) & $\mathrm{X}$ & \\
\hline $\begin{array}{l}\text { PAB per capita dos municípios não-habilitados acrescidos } \\
\text { de Fração Assistencial Especializada (Gestão Avançada do } \\
\text { Sistema Estadual — GASE) }\end{array}$ & $\mathrm{X}$ & \\
\hline Teto financeiro global de estado (GPSE) & $\mathrm{X}$ & \\
\hline Incentivo financeiro do PACS/PSF & $\mathrm{X}$ & \\
\hline $\begin{array}{l}\text { Teto financeiro de epidemiologia e controle de doenças } \\
\text { (estados e municípios habilitados) }\end{array}$ & & $\mathrm{X}$ \\
\hline $\begin{array}{l}\text { Piso Básico de Vigilância sanitária per capita } \\
\text { (estados e municípios habilitados) }\end{array}$ & $\mathrm{X}$ & \\
\hline $\begin{array}{l}\text { Índice de Valorização de Impacto em Vigilância Sanitária } \\
\text { (estados habilitados) }\end{array}$ & & $\mathrm{X}$ \\
\hline Índice de Valorização de Resultados (estados habilitados) & & $\mathrm{X}$ \\
\hline $\begin{array}{l}\text { Incentivo de combate às carências nutricionais per capita } \\
\text { (municípios habilitados situados abaixo da linha } \\
\text { de pobreza definida pelo IPEA) }\end{array}$ & $\mathrm{X}$ & \\
\hline $\begin{array}{l}\text { Incentivos para farmácia básica (municípios habilitados } \\
\text { com população inferior a } 21.000 \text { habitantes) }\end{array}$ & $\mathrm{X}$ & \\
\hline
\end{tabular}

Fonte: Levcovitz, E. Seminário “Separação Financiamento/Provisão de Serviços”. Ministério da Saúde/ Banco Mundial. Rio de Janeiro, Abril 1999.

A meta da NOB 1/96 para o final de 1998 era a transferência ao total de 3.300 municípios da responsabilidade pela assistência básica, bem como habilitar 700 outros na gestão plena dos respectivos sistemas 
locais de saúde. No primeiro caso, a meta foi ultrapassada em $41 \%$ já no fim do segundo semestre de 1998. No segundo caso, o grau de comprometimento da meta alcançava $67 \%$ em novembro de 1998 , o que significa que $93 \%$ (cerca de 5.136) dos municípios brasileiros passaram a exercer o comando de seus serviços de saúde, principalmente os de atenção primária, conforme pode ser visto na tabela abaixo:

Tabela 8: Municípios habilitados segundo a condição de gestão por região. Brasil, 1998

\begin{tabular}{|c|c|c|c|c|c|}
\hline \multirow[b]{2}{*}{ Estados } & \multirow{2}{*}{$\begin{array}{c}\text { Total } \\
\text { de } \\
\text { Municípios }\end{array}$} & \multicolumn{3}{|c|}{ Pleitos aprovados por condição de gestão } & \multirow[b]{2}{*}{$\%$} \\
\hline & & GPAB* & GPSM $* * 1$ & Total & \\
\hline Norte & 449 & 341 & 46 & 387 & 86,2 \\
\hline Nordeste & 1.787 & 1.548 & 97 & 1.645 & 92,1 \\
\hline Sudeste & 1.666 & 1.304 & 267 & 1.571 & 94,3 \\
\hline Centro-Oeste & 446 & 425 & 19 & 444 & 99,8 \\
\hline Sul & 1.159 & 1.047 & 42 & 1.085 & 94,0 \\
\hline Brasil & 5.506 & 4.665 & 471 & 5.136 & 93,3 \\
\hline
\end{tabular}

Fonte: MS - Secretaria de Políticas de Saúde/Coordenação de Implementação da Descentralização.

*Gestão Plena da Assistência Básica.

**1 Gestão Plena do Sistema Municipal, posição em 31.12.98

Nos primeiros seis meses de 1998, o MS já havia transferido para 4.664 municípios, habilitados nas duas formas de gestão descentralizada, recursos correspondentes a cerca de $30 \%$ do dispêndio federal com assistência médico-ambulatorial no mesmo período, conforme demonstrado na tabela abaixo:

Tabela 9: Recursos repassados pelo MS, por modalidade de gestão descentralizada. Brasil

\begin{tabular}{l|c|c}
\hline \multirow{2}{*}{ Modalidades de gestão } & \multicolumn{2}{|c}{ Recursos transferidos } \\
\cline { 2 - 3 } & Em R\$1.000,00 & $\%$ \\
\hline Gestão Plena da Assist. Básica & 328.263 & 32,3 \\
\hline Gestão Plena do Sistema de Saúde & 690.335 & 67,7 \\
\hline Total & 1.018 .598 & 100 \\
\hline
\end{tabular}

Fonte: MS - Secretaria de Políticas de Saúde/Coordenação de Implementação da Descentralização.

Tomando-se apenas os 471 municípios habilitados na GPSM, a Tabela 10 mostra que, no ano de 1998, eles executaram $41 \%$ do orçamento do MS para prestação de serviços. 
Tabela 10: Execução do orçamento

federal, segundo o tipo de prestador.

Brasil, 1998

\begin{tabular}{l|c|c}
\hline Tipo de prestador & Valor executado (R\$ 1,00) & Participação (\%) \\
\hline Federal & 50.278 .419 & 0,60 \\
\hline Estadual & 791.308 .254 & 9,5 \\
\hline Municipal & 633.932 .312 & 7,6 \\
\hline Privado Contratado & 1.218 .124 .590 & 14,7 \\
\hline Filantrópico & 1.185 .557 .009 & 14,3 \\
\hline Universitário & 997.898 .260 & 12,1 \\
\hline Sindicato & 6.561 .368 & 0,09 \\
\hline Gestão Plena Municipal & 3.389 .946 .195 & 41,0 \\
\hline Total & 8.273 .606 .410 & 100 \\
\hline
\end{tabular}

Fonte: MS — Datasus, 1999.

Esse processo acelerado de adesão à descentralização, ocorrido no ano de 1998, é explicável pela natureza distributiva da NOB 1/96 ao propor, inicialmente, a média do $\mathrm{PAB}$ per capita de $\mathrm{R} \$ 1,00$ à nova dinâmica de habilitação, produziu a seguinte situação:

- $66 \%$ dos municípios (3.243) tinham o PAB per capita menor que $\mathrm{R} \$ 1,00 \mathrm{e}$, portanto, ganharam recursos financeiros com o PAB de R \$ 1,00;

- 22\% dos municípios (1.078) tinham um PAB per capita entre $\mathrm{R} \$ 1,00$ e $\mathrm{R} \$ 1,50$; portanto, não perderam nem ganharam recursos financeiros, porque se habilitavam a receber o fator diferenciado de ajuste à implantação do $\mathrm{PAB}$, no valor máximo de até $\mathrm{R} \$ 0,50$ por habitante;

- $12 \%$ dos municípios (573) tinham um PAB per capita acima de $\mathrm{R} \$ 1,50$ e, portanto, tiveram perdas no valor que excedia a esse valor.

Os gestores dos estados de maior capacidade de arrecadação de recursos do orçamento da saúde pelo critério de capacidade instalada, especialmente São Paulo, Minas Gerais, Rio de Janeiro e Rio Grande do Sul vetaram, inicialmente, a proposta distributiva da NOB 1/96, porque essa favoreceu os estados e municipalidades do Norte e Nordeste. Esse ponto de veto é uma explicação plausível para a demora de implementação da NOB1/96 e para a paralisação da descentralização ao longo do ano de 1997 (MS, 1997a). 
A proposta de distribuição pura e simples dos recursos do PAB per capita altera a lógica de distribuição territorial ainda vigente de financiamento de internação hospitalar e assistência ambulatorial, baseada na capacidade de oferta já instalada. A mudança do critério distributivo para um critério populacional coloca recursos do SUS em municípios sem oferta ou rede estruturada. Esse esforço distributivo pode tanto resultar em uma política pulverizadora de recursos, como também um efeito indutivo e virtuoso de maior responsabilização das municipalidades beneficiadas.

A NOB 1/96 introduz, como inovação relevante, uma lista de estímulos financeiros à adoção de programas de atenção básica municipal e incentivos aos PSF e PACS, buscando mudar o enfoque clínico da atenção à saúde centrado na doença e no indivíduo, para um enfoque centrado no ambiente social e familiar, recorrendo à estratégia de promoção da saúde. Além do que, a NOB 1/96 oferece o PAB como uma oferta mínima homogênea ou cesta básica para todo o país.

Ao contrário do que tem se verificado para os municípios, ainda é pouco significativa a adesão dos estados ao novo papel que lhes foi reservado no SUS. O papel dos governos estaduais foi revisto pela NOB 1/96, que prevê, para essas instâncias duas modalidades de gestão:

- Gestão Avançada do Sistema Estadual (GASE);

- Gestão Plena do Sistema Estadual (GPSE).

Para a habilitação das secretarias de saúde a essas duas condições de gestão são demandadas, entre outras exigências:

- Plano estadual de saúde;

- Programação pactuada integrada;

- Plano estadual de prioridade de investimentos;

- Plano de gerência de unidades estatais de hemorrede e de laboratório de referência para controle de qualidade, vigilância sanitária e vigilância epidemiológica;

- Proposta de organização de sistemas de referência e de câmara de compensação de autorizações de internação hospitalar, procedimentos especializados e de alto custo ou alta complexidade.

Para o estado obter a condição inicial de GASE, exige-se que disponha de $80 \%$ dos municípios em condições de habilitação em uma das duas das condições da NOB 1/96. Em dezembro de 1998, apenas os Estados do Acre, Amazônia, Amapá e Roraima não preenchiam esse requisito, conforme tabela a seguir. 
Tabela 11: Distribuição dos municípios habilitados por estado segundo a NOB 1/96. Brasil

\begin{tabular}{|c|c|c|c|c|}
\hline Estado & $\begin{array}{c}\text { Total de } \\
\text { municípios }\end{array}$ & $\begin{array}{c}\text { \% Gestão } \\
\text { Plena da } \\
\text { Atenção Básica }\end{array}$ & $\begin{array}{l}\text { \% Gestão Plena } \\
\text { do Sistema } \\
\text { Municipal }\end{array}$ & $\begin{array}{c}\text { \% de municípios } \\
\text { habilitados } \\
\text { pela NOB } 1 / 96\end{array}$ \\
\hline $\mathrm{AC}$ & 22 & 68,18 & 4,55 & 72,73 \\
\hline $\mathrm{AL}$ & 101 & 90,10 & 9,90 & 100,00 \\
\hline $\mathrm{AM}$ & 62 & 62,90 & 1,61 & 64,52 \\
\hline AP & 16 & 43,75 & 0,00 & 43,75 \\
\hline BA & 415 & 87,23 & 1,93 & 89,16 \\
\hline $\mathrm{CE}$ & 184 & 78,80 & 14,67 & 93,48 \\
\hline ES & 77 & 36,36 & 55,84 & 92,21 \\
\hline GO & 242 & 95,45 & 4,13 & 99,59 \\
\hline MA & 217 & 75,58 & 15,21 & 90,78 \\
\hline MG & 853 & 88,86 & 5,74 & 94,61 \\
\hline MS & 77 & 89,61 & 10,39 & 100,00 \\
\hline MT & 126 & 99,21 & 0,79 & 100,00 \\
\hline PA & 143 & 67,83 & 19,58 & 87,41 \\
\hline $\mathrm{PB}$ & 223 & 87,89 & 0,90 & 88,79 \\
\hline $\mathrm{PE}$ & 185 & 96,22 & 3,78 & 100,00 \\
\hline PI & 221 & 83,26 & 0,45 & 83,71 \\
\hline PR & 399 & 96,74 & 3,26 & 100,00 \\
\hline RJ & 91 & 80,22 & 19,78 & 100,00 \\
\hline RN & 166 & 92,77 & 5,42 & 98,19 \\
\hline RO & 52 & 76,92 & 23,08 & 100,00 \\
\hline $\mathrm{RR}$ & 15 & 60,00 & 0,00 & 60,00 \\
\hline RS & 467 & 85,22 & 1,50 & 86,72 \\
\hline $\mathrm{SC}$ & 293 & 89,76 & 7,51 & 97,27 \\
\hline $\mathrm{SE}$ & 75 & 98,67 & 0,00 & 98,67 \\
\hline SP & 645 & 68,99 & 24,34 & 93,33 \\
\hline TO & 139 & 96,40 & 2,88 & 99,28 \\
\hline Brasil & 5506 & 84,73 & 8,55 & 93,28 \\
\hline
\end{tabular}

Fonte: MS/SPS - Secretaria de Políticas de Saúde/Coordenação da Implantação da Descentralização.

Posição em 15/12/1998. 
Apesar dos indicadores de desempenho municipal demonstrados, apenas o Estado de Alagoas obteve a habilitação na condição plena do sistema estadual até 31 dezembro de $1998 .{ }^{3}$ No primeiro trimestre de 1999, demandaram a habilitação na condição de GPSE os Estados de Minas Gerais, Paraná e Santa Catarina. Os Estados da Bahia, Rio Grande do Sul e São Paulo apresentaram pleito para obter a condição de GASE.

Esse processo retardatário e desigual sinaliza as dificuldades dos estados em definirem um papel claro na estrutura do sistema público de saúde brasileiro, dominada ainda, na década de 90, pelas demandas e orientações localistas. Essa situação tem sido comprovada por conclusões oriundas de pesquisa empírica. Assim, por um lado, o gestor estadual é o que sofre o maior impacto do processo de descentralização ao ter seu papel original de executor de ações, voltadas para o atendimento da população e de contratante de serviços privados e filantrópicos, substituído por uma função de coordenação, apoio e regulação do sistema estadual de saúde. Por outro, as prerrogativas das secretarias estaduais de saúde em termos de coordenação, articulação e estruturação do sistema de saúde intermunicipal ou regional apresentam uma baixa institucionalidade (MS, 1997b: Relatório de gestão). Da mesma forma, a ausência de parceria entre gestores municipais e as secretarias estaduais de saúde para o desenvolvimento de ações de cooperação técnica tem levado as secretarias municipais à contratação dessas nas universidades e organizações não-governamentais, para suprir necessidades de treinamento e de desenvolvimento de novos métodos de gestão (Instituto de Saúde, 1998: 64).

O Banco Mundial sugere um conjunto de ações em nível estadual que podem fortalecer as suas funções regionais, hoje eclipsadas pela capacidade de formulação e organização da agenda das instâncias federal e municipais, e que são as seguintes:

- desenvolver um modelo eqüitativo de distribuição entre municipalidades, ajustando-as aos modelos de distribuição per capita e de capacidade de produção de serviço;

- fortalecer a utilização compartilhada de serviços e especializações de referências entre municípios, promovendo economia de escala e estimulando as oportunidades de especialização municipal; as iniciativas dos consórcios intermunicipais de saúde seriam exemplos desse esforço regional;

- estimular a definição de prioridades nas intervenções de saúde;

- estabelecer combinação de preços, orçamento global e outros instrumentos para a definição de prioridades pelos gestores locais, considerando que as transferências intragovernamentais tendem a ser de fundo a fundo;

- estudar a ineficiência alocativa devido a ausência de estratégias de tratamento preventivo; 
- planejar os investimentos futuros em incorporação tecnológica e equipamentos, de acordo com as novas funções na gestão de custos do Governo Federal e estados;

- estimular as organizações e serviços públicos a serem mais autônomos e responsabilizáveis (accountable), introduzindo mecanismos de flexibilidade nas relações de trabalho, contratos de gestão e competição para a prestação de serviços específicos demandados pelo gestor (World Bank, 1999).

O MS tem sinalizado, desde os primeiros meses de 1999, que, em decorrência do aumento da autonomia de gestão municipal, cobrará resultados baseados em metas da atenção básica à saúde. Pela proposta aprovada pela CIT, todos os 5.136 municípios habilitados pela NOB 1/96 deverão apresentar resultados em 17 itens de desempenho associados à provisão de atenção básica, que incluem cobertura vacinal, mortalidade infantil, número de hospitalizações por tétano, sarampo, difteria, etc.

Os estados e municípios tiveram prazo até 30/05/99 para definirem as metas a serem atingidas em cada atividade. Os resultados propostos deverão ser atingidos até dezembro, quando serão analisados pelo Governo Federal. O MS desenvolverá mecanismos de recompensa para quem melhorar seus índices e punição para aqueles que não conseguirem cumprir os objetivos estabelecidos. Por esse instrumento, o MS poderá reconhecer que a descentralização foi implantada com deficiência na fiscalização e no controle da aplicação dos recursos. O Governo Federal ainda não teve sucesso na elaboração de um sistema preciso de informações estatísticas sobre saúde associado ao desempenho da gestão pública (Gazeta Mercantil, 05/03/1999).

\section{Conclusões: balanço e perspectivas}

São poucas as evidências de que a descentralização setorial tenha afetado de modo negativo a qualidade dos serviços de saúde, negligenciando o desenvolvimento de mecanismos de responsabilização dos governos locais, ou até mesmo que tenha gerado um processo descoordenado, caótico e/ou produzido uma crise de legitimidade do sistema público de saúde.

Como também são poucas as evidências de que a crescente responsabilização pública local pela gestão da política de saúde seja apenas um resultado direto da barganha federativa, segundo a definição de Arretche (1998). Segundo esse autor, dadas as dimensões da pobreza brasileira e, portanto, da população-alvo dos programas sociais, a gestão de políticas sociais tenderia a ser, simultaneamente, cara e inefetiva, na medida em 
que consumiria um elevado volume de recursos com baixos níveis de proteção efetiva. Nesse caso, nas situações em que os custos políticos e/ou financeiros da gestão de uma dada política forem avaliados como muito elevados, a barganha federativa consiste em buscar atribuí-los ou imputálos a um outro nível de governo (op.cit.: 36).

Os primeiros balanços sobre o impacto da implementação da NOB 1/93, especialmente nos municípios habilitados na condição semiplena, indicam que essa condição de gestão:

- projeta as secretarias municipais de saúde na esfera dos governos municipais;

- amplia a capacidade de gestão em função das novas responsabilidades;

- aumenta a força de trabalho na saúde;

- gera aumento na oferta de serviços ambulatoriais e ações de saúde coletiva;

- induz a um maior comprometimento de recursos do orçamento municipal com o setor saúde (Instituto de Saúde, 1998).

A pesquisa da Unicef/Gallup (1996) destinada a medir a satisfação com o nível dos serviços públicos de atendimento à saúde da população foi também favorável aos gestores locais, em situação de gestão semiplena. A pesquisa entrevistou indivíduos da população adulta (18 anos ou mais), residentes em área urbana, de 42 municípios discriminados em com e sem gestão semiplena. As respostas dos usuários dos serviços de saúde favoreceram os municípios em situação de gestão semiplena em comparação aos demais:

- pela maior satisfação na melhoria nos serviços;

- pela maior percepção de solução dos problemas;

- pela maior aprovação da qualidade dos serviços;

- pela atribuição de notas mais elevadas aos serviços;

- pela maior presteza no atendimento;

- pela menor falta de médicos e equipamentos;

- pela melhor imagem pública dos serviços.

Esses resultados parciais favoráveis ao processo de descentralização também foram ratificados pela pesquisa geral em amostra domiciliar realizada pelo Conselho Nacional de Secretários Estaduais de Saúde, Fundação Nacional de Saúde e Ibope, em 1998, que inquiriu sobre a satisfação dos brasileiros com os serviços públicos de saúde. Os resultados foram:

- $76 \%$ dos usuários de estabelecimento público de saúde afirmam que foram bem atendidos;

- $75 \%$ consideram o atendimento médico satisfatório na rede pública;

- $59 \%$ consideram satisfatória a rede pública em geral.

Não deixa de ser ainda um assunto relevante para o processo de descentralização a criação de mecanismos de avaliação autônomos e 
sistemáticos da reforma descentralizadora do setor saúde, em função de um conjunto de riscos:

- a baixa capacidade administrativa dos municípios na área de saúde;

- o uso das transferências SUS como simples reforço para o orçamento municipal ou para outros fins que não a saúde;

- a atomização e a pulverização dos serviços de saúde decorrentes da tendência dos municípios procurarem ter seu próprio hospital ou serviços;

- a ausência de cultura de gestão orientada para a economia de escala ou pela utilização de sistema de referência apropriado (World Bank, 1999: 51; Lucchese, 1996).

Vianna e Piola sugerem, numa perspectiva otimista sobre o impacto da descentralização, que um dos efeitos relevantes introduzidos pela NOB 1/96 foi a ampliação da responsabilidade dos gestores estaduais e locais em relação aos recursos federais que lhes são transferidos. As antigas modalidades incipientes e parciais da NOB 1/93, embora incentivassem o aumento da produção de serviços, criavam expectativas e pressões por mais recursos federais ao fortalecer, nas instâncias subnacionais, a cultura do provedor privado remunerado segundo a quantidade e densidade tecnológica de procedimentos.

Ao contrário, quando enquadrado em quaisquer das duas modalidades de gestão plena pela NOB 1/96, ficam bem evidentes os limites dos repasses federais face às necessidades de financiamento do sistema local, o que gera "...um incentivo natural à contenção de custos, ao uso de recursos próprios e uma maior responsabilidade pela gestão dos serviços"(Vianna \& Piola, 1999:12).

Muitas das interrogações dos autores sobre os efeitos da descentralização valeriam como motivação para um segundo movimento de reforma do setor saúde e das funções do Estado brasileiro na provisão de serviços sociais.

As incertezas sobre eficiência e efetividade da descentralização aparecem associadas a dois problemas centrais: a) o papel das instâncias mesorregionais; b) a reduzida capacidade de controle dos cidadãos/usuários sobre as decisões dos administradores públicos.

Em relação ao primeiro problema, as incertezas sinalizadas estimulam a busca de modelos centrados no fortalecimento da autoridade governamental estadual ou de associações regionais livres entre municípios de pequeno porte. A percepção dos problemas ocasionados pela municipalização sob baixa regulação gerou a proposta de correção da perda de capacidade de coordenação, mediante a criação de sistemas microrregionais de serviços de saúde (Vilaça Mendes, 1998). O problema central nessa abordagem está em descartar a livre associação entre os municípios, por meio dos consórcios, que são considerados como um modelo ineficiente 
e de baixa sustentabilidade. A proposta desconsidera, totalmente, os ganhos que a cooperação entre gestores traz para a governabilidade da coordenação supralocal.

Outra solução experimentada no passado destacou as funções das secretarias estaduais. A regionalização coordenada pela esfera estadual consistiu em um modelo organizacional composto de diversas secretarias estaduais de saúde, nos anos 80, reunidas em escritórios regionais que geravam alta subordinação à autoridade municipal. $\mathrm{O}$ próprio Instituto Nacional de Assistência Médica da Previdência Social (INAMPS), criado em 1974, apresentava, através das superintendências estaduais, um modelo centralizado baseado na padronização dos procedimentos, porém com elevado grau de autoridade regional. $\mathrm{O}$ modelo regionalizado da atenção pública envolvia relações baseadas em convênios estabelecidas caso a caso entre União e/ou estados com os municípios.

O terceiro modelo em disponibilidade para a coordenação mesorregional — os consórcios intermunicipais de saúde — apresenta algumas vantagens comparativas:

- pertence à tradição recente do SUS e se disseminou por todo o País;

- oferece expressivo ganho em escala com relação à utilização de leitos hospitalares e serviços médicos especializados, além da utilização de recursos diagnósticos de alto custo;

- exibe experiências exitosas de elevada sustentabilidade;

- gera parcerias não-compulsórias;

- estimula relações cooperativas que transcendem a política de saúde;

- estabelece cooperação com as secretarias estaduais cada vez mais freqüentes;

- pode permitir alta flexibilidade na gestão sem perda do caráter público, caso a sociedade civil criada seja regulada por um conselho curador;

- permite a implementação da descentralização por agências regionais.

As desvantagens usualmente observadas nos consórcios decorrem das condições locais, na medida em que o sucesso da associação é fortemente dependente da capacidade política de desenvolver parcerias e redistribuir o bônus da cooperação:

- muitos consórcios objetivam, simplesmente, dotar a rede de determinados especialistas médicos;

- a cooperação política envolve muitas vezes elevados custos de transação;

- muitas parcerias, especialmente as decorrentes de indução ou determinação estadual, mostraram baixa sustentabilidade;

- o objetivo de regular a atenção à saúde na região é pouco compartilhado, mostrando baixa utilização dos ganhos potenciais da parceria; 
- em regiões mais pobres, o êxito na ampliação do escopo dos consórcios e seu impacto depende de investimentos da esfera estadual.

A reestadualização do sistema não aparece como uma forte tendência no SUS, apesar da presença de diversos secretários estaduais com tradição setorial e compromissos de governadores com vigorosa ampliação dos gastos estaduais em saúde. Podemos esperar que, assim como o MS, que representa o principal financiador do sistema, os governos estaduais devam ampliar a sua capacidade regulatória sobre o desempenho municipal, conforme maior comprometimento de seus recursos financeiros. Um desfecho possível é a regionalização desenhada a partir dos estados, sendo esperadas reações dos municípios à perda de autonomia e o crescimento das soluções definidas localmente em resposta aos problemas de eficiência sistêmica, mediante a adoção de modelos de livre associação - os consórcios.

Quanto ao problema da reduzida capacidade de controle dos cidadãos/usuários sobre as decisões dos administradores públicos, cabe assinalar que o insulamento burocrático, o formalismo e a alta autonomia têm contribuído para a estagnação da administração pública bastante reativa às novas formas de gestão - essa continua voltada ao controle de procedimentos e não de resultados, e é pouco sensível ao julgamento dos usuários sobre a qualidade dos bens e serviços produzidos.

Essa combinação pode ameaçar a boa implementação dos programas de saúde, por não inibir as condutas oportunistas. Nesse modelo de gestão estão ausentes os mecanismos de responsabilização (accountability) sobre as decisões e escolhas dos gestores. A agenda orientada para a qualidade e responsabilidade na gestão pública, no contexto da descentralização, tem como desafio responder por um novo desenho institucional baseado na contratualização vis-à-vis a autonomia de gestão no plano organizacional.

É certo que os avanços verificados na ampliação do federalismo sanitário estarão sob alto risco, caso os custos de transação gerados pela descentralização (aumento do número de contratantes e agentes) não assegurem, pelo contrário, dissipem recursos e não apresentem benefícios sustentáveis para o cidadão/usuário do sistema público, como verificado em países com trajetória similar, sendo a Itália o melhor exemplo (Borgovoni \& Pintus, 1996).

A experiência internacional de reforma do setor saúde oferece alternativas organizacionais consideráveis para transformar a capacidade do Estado em situações de elevada incerteza, face aos resultados de uma reorientação de política pública, como é o caso da descentralização. $\mathrm{O}$ ambiente de incerteza permite, principalmente, inovar frente os modelos de gestão centrados no insulamento e na baixa responsabilização dos agentes públicos. Uma dessas alternativas é a proposta de contratualização, 
que diversifica e amplia os mecanismos de responsabilização das organizações públicas. Nesse sentido, o contrato de gestão, como uma orientação estratégica para toda a administração pública, pode apontar para a institucionalização de mecanismos negociados de regulação e orientação para a qualidade.

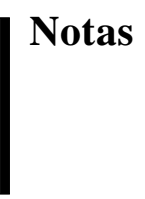

${ }^{1}$ Este trabalho foi desenvolvido no Projeto Configuração Econômica do Setor Saúde Nepp/Unicamp e Instituto de Economia — Unicamp, junho de 1999.

2 Mantendo os demais instrumentos de pagamento para os prestadores de atenção hospitalar - Sistema de Informações Hospitalares do SUS (AIH/SUS); e de transferência para os municípios e estados de recursos para pagamento de cuidado ambulatorial — Sistema de Informações Ambulatoriais (SIA/SUS).

3 O Distrito Federal, por razões peculiares, obteve a habilitação na GASE ainda no ano de 1998.

\section{Referências bibliográficas}

Afronso, R. B. A. \& SILVA, P. L. B. (1995), A Federação em Perspectiva. Ensaios Selecionados. São Paulo, Fundap.

Afonso, J. R. R. et al. (1999), "Municípios, Arrecadação e Administração Tributária: Quebrando Tabus". Rio de Janeiro, BNDES, p.6

Arretche, M. T. S. (1998), "O Processo de Descentralização das Políticas Sociais no Brasil". Tese de Doutorado/ Instituto de Filosofia e Ciências Humanas/Unicamp.

Borgovoni, E \& Pintus, E. (1996), Rethinking the National Health System. The Level of decentralization after the 1992 reforma law. Milan: Cergas/Bocconi University.

CONASS/FNS/IBOPE. (1998), Promoção da Saúde - Pesquisa de Opinião. Brasília.

Gazeta Mercantil. (1999), "Governo Estabelece Metas Para a Saúde”. 05/03, p.A-14.

IBGE. (1993). Pesquisa Assistência Médico-Sanitário no Brasil. Rio de Janeiro.

Instituto de SAÚde. (1998), "A Descentralização do Sistema de Saúde no Brasil: uma proposta de investigação: Relatório Final”. São Paulo.

Lucchese. P. T. R. (1996), Descentralização do Financiamento e Gestão da Assistência à Saúde no Brasil: A Implementação do Sistema Único de Saúde (1990-1995) Planejamento e Políticas Públicas (14), Brasília: Ipea.

MinISTÉRIo da SAÚde. (1997a), Novos Estudos sobre os Tetos Financeiros para a Assistência à Saúde e Vigilância Sanitária para 1988 - Brasil e por Estado. Brasília.

Ministério da SAÚde. (1997b), Avaliação da Gestão da Assistência Ambulatorial e Hospitalar do SUS. Brasília. 
Ministério da SAÚde. (1997c), Secretária de Políticas/Avaliação da Descentralização. Brasília

PNUD. ( 1996), Relatório sobre o Desenvolvimento Humano no Brasil. Brasília: Ipea.

RePública Federativa do Brasil. (1988), Constituição de 1988.

Unicef/Instituto Gallup. (1996), Pesquisa de Opinião Pública. Brasília.

Via I Redons, J. M. \& Mcdonagh, F. G. (1999), "Decentralization in the Health Sector". World Bank (Paper prepared for the Annual World Bank Conference on Development in Latin America and the Caribbean).

Vianna, S. M. \& Piola, S. F. (1999), "Descentralização e Gestão do Gasto Público com Saúde no Brasil". Texto Produzido para o XI Seminário Regional de Política Fiscal. Cepal/Ipea.

Vilaça Mendes, E. (org.) (1998), A Organização da Saúde no Nível Local. São Paulo: Hucitec.

World BAnk. (1998), Brazil - Fiscal Adjustment and Social Spending. The Case of Education and Health in Four Brazilian States. Washington.

World Bank. (1988), Brazil Public Spending on Social Programs: Issues and Options. Washington. 


\section{A descentralização do sistema de saúde no Brasil}

\section{Nilson do Rosário Costa, Pedro Luís Barros Silva e José Mendes Ribeiro}

Os autores abordam a experiência brasileira de descentralização do sistema público de saúde, representada pela criação do Sistema Único de Saúde (SUS), a partir da Constituição de 1988, e a sua operacionalização mediante as Normas Básicas Operacionais ((NOBs). Foram redefinidos radicalmente os papéis da União, dos estados e municípios no que se refere à oferta de serviços de atenção à saúde, e, por meio da análise de um conjunto variado de dados, são feitas não só a descrição do sistema em funcionamento, como a verificação de vantagens e desvantagens ocorridas, além de sugestões que poderão contribuir ao aperfeiçoamento do modelo implantado.

\section{La descentralización del sistema de salud en Brasil}

Nilson do Rosário Costa, Pedro Luís Barros Silva y José Mendes Ribeiro

Los autores abordan la experiencia brasileña de descentralización del sistema público de salud, representada por la creación del Sistema Único de Salud (SUS) a partir de la Constitución de 1988, y su operalización mendiante las Normas Básicas Operacionales (NOBs). Se redefinieron radicalmente los roles de la Federación y de los estados y municipios en lo que se refeiere a la oferta de servicios de atención en el área de salud y, a través del análisis de un amplio conjunto da datos, se describe el sistema en funcionamiento, se definen las ventajas y desventajas verificadas, y se presentan sugerencias que podrían contribuir con el perfeccionamiento del modelo implantado.

\section{Decentralisation of the health system in Brazil}

\section{Nilson do Rosário Costa, Pedro Luís Barros and José Mendes Ribeiro}

The authors address the Brazilian experience in decentralisation of the public health system, represented by the establishment of the Single Health System (SUS), as from the enactment of the 1988 Constitution, and by its operationalisation by means of the Basic Operational Norms (NOBs). The roles of the Union, of the States and Municipalities were radically redefined as far as the supply of health-care services is concerned and, by means of the analysis of a varied set of data, they carry out not only the description of systems in operation, but also the verification of advantages and disadvantages incurred, in addition to making suggestions that may contribute to the improvement of the model put in place.

Contato com o autor: nilsonrc@unisys.com.br pbar@nepp.unicamp.br jmribeiro@ax.apc.org
Revista do

Serviço

Público

Ano 50

Número 3

Jul-Set 1999

Nilson do Rosário Costa, sociólogo, doutor em planejamento urbano e pesquisador titular da ENSP/ FIOCRUZ/RJ.

Pedro Luís Barros Silva, administrador público, doutor em ciência política, professor do Instituto de Economia e coordenador do NEPP/ UNICAMP/SP. José Mendes Ribeiro, médico, doutor em ciências e pesquisador adjunto da ENSP/ FIOCRUZ/RJ 